\title{
Bounded countable atomic compactness of ordered groups
}

\author{
by
}

\author{
Friedrich Wehrung (Caen)
}

\begin{abstract}
We show that whenever $A$ is a monotone $\sigma$-complete dimension group, then $A^{+} \cup\{\infty\}$ is countably equationally compact, and we show how this property can supply the necessary amount of completeness in several kinds of problems. In particular, if $A$ is a countable dimension group and $E$ is a monotone $\sigma$-complete dimension group, then the ordered group of all relatively bounded homomorphisms from $A$ to $E$ is a monotone $\sigma$-complete dimension group.
\end{abstract}

0. Introduction. By definition, an ordered abelian group is monotone $\sigma$-complete whenever every bounded increasing sequence of elements admits a l.u.b. We will here be concerned with those monotone $\sigma$-complete groups that are in addition directed and satisfy the Riesz interpolation property. One can then show that such groups are (strongly) Archimedean, thus they are dimension groups $[7,8]$. Monotone $\sigma$-complete dimension groups appear naturally as ordered Grothendieck groups $K_{0}$ of countably continuous regular rings and thus intervene also in the study of Rickart $\mathrm{C}^{*}$-algebras $[7,8,9]$.

Intriguingly, there is another related large class of ordered structures, the class of Tarski's cardinal algebras, as well as other more general classes, as e.g. refinement algebras [14]. Basically, these objects also appear naturally in a lot of cases as algebras of isomorphism types of various structures such as sets under equipotence or $\sigma$-complete Boolean algebras. Many of their properties are already valid in the more general class of weak cardinal algebras, defined simultaneously and independently by K. P. S. Bhaskara Rao and R. M. Shortt on the one hand, and the author on the other hand in [11, $18,19]$.

1991 Mathematics Subject Classification: Primary 06F05, 06F20, 08A45; Secondary 19A49, $19 \mathrm{~K} 14$.

Key words and phrases: monotone $\sigma$-complete groups, dimension groups, equational compactness. 
Then it is not difficult to verify that if $A$ is an abelian ordered group, then $A^{+} \cup\{\infty\}$ is a weak cardinal algebra if and only if $A$ is monotone $\sigma$-complete and satisfies the finite interpolation property, and this gives a hint that methods used for one theory can often be used for the other. For example, norm-completeness of Archimedean abelian groups with unit with the countable interpolation property [7] is very closely related to metric completeness of weak cardinal algebras and more general objects [19].

There is another striking common point between the latter two results; it is that they are particular cases of atomic compactness [1, 10, 15, 16, 17]. Since most natural ordered groups fail to enjoy any topological compactness property under some intrinsic topology (like e.g. the order topology), atomic compactness could be the best one can hope for in that direction. Furthermore, there are several other positive results of that flavor:

- The characterization in [18] of injective positively ordered monoids.

- The result that divisible weak cardinal algebras (and more general objects) are countably injective, thus countably atomic compact [20]. In particular, if $A$ is any divisible (not necessarily Archimedean) dimension group with the countable interpolation property, then $A^{+} \cup\{\infty\}$ is countably injective.

- The result that if $A$ is a Dedekind complete $f$-ring, then $(A,+,-, \cdot, \wedge$, $\vee, \leq)$ is "boundedly atomic compact" [21, Theorem 5.5].

- The more recent result [22] that if $A$ is a Dedekind $\sigma$-complete $f$-ring, then $(A,+,-, \cdot, \wedge, \vee, \leq)$ is "boundedly countably atomic compact".

In this paper, we generalize the latter result to monotone $\sigma$-complete dimension groups. The proof we present here does not use any forcing, but is valid only for the language $(+, \leq)$.

In Section 1, we recall the classical definitions and terminology about atomic compactness $[1,10,15,16]$, and we introduce bounded atomic compactness, which (roughly speaking) is to atomic compactness as local compactness is to compactness in general topology. Such a situation has already been encountered in [21, Theorem 5.5] and [22].

In Section 2, we present a proof of the fact that every monotone $\sigma$ complete dimension group is boundedly countably atomic compact in the language $(+, \leq)$ (Theorem 2.9); unlike the proofs of atomic compactness in $[21,22]$, it does not use any forcing, relying instead on the analysis of the structure of the set of solutions of a given finite linear system with parameters from the group in question. It is our belief that there are even stronger forms of compactness satisfied by monotone $\sigma$-complete dimension groups to be investigated yet. Furthermore, in Theorem 2.11, we prove an algebraic analogue of the classical topological "closed projection theorem" (if $K$ is compact, then the natural projection from $X \times K$ to $X$ is closed). 
In Section 3, we show how bounded countable compactness can be applied to generalize some abstract measure extension problems (Proposition 3.1) and a Hahn-Banach like theorem for monotone $\sigma$-complete dimension groups (Theorem 3.4). Some of these results were known in the lattice case, but without the possibility to take countable suprema, atomic compactness properties are just what is needed to overcome this difficulty.

If $X$ and $Y$ are two sets, then ${ }^{X} Y$ will denote the set of all maps from $X$ to $Y$.

We will denote the set of all positive integers by $\omega$ or $\mathbb{N}$, according to whether we consider it as an ordinal or a monoid; in the first case, recall that if $n$ is a positive integer, then $n=\{x: x<n\}$.

Let $(P, \leq)$ be an ordered set. For all $a, b$ in $P,[a, b]$ will denote the interval $\{x \in P: a \leq x \leq b\}$. If $X, Y$ are two subsets of $P$, then $X \leq Y$ will stand for $(\forall x \in X)(\forall y \in Y)(x \leq y)$; if $X=\{a\}$ (resp. $Y=\{a\}$ ), we will write $a \leq Y$ (resp. $X \leq a)$; if $X=\left\{a_{1}, \ldots, a_{m}\right\}$ and $Y=\left\{b_{1}, \ldots, b_{n}\right\}$, we will also write $a_{1}, \ldots, a_{m} \leq b_{1}, \ldots, b_{n}$. We will say that $P$ is directed (resp. filtered) when for all $x, y$ in $P$, there exists $z$ in $P$ such that $x, y \leq z$ (resp. $z \leq x, y$ ). If $x, x_{n}$ $(n \in \omega)$ are elements of $P$, say that $x=\bigvee_{n} \uparrow x_{n}$ (resp. $\left.x=\bigwedge_{n} \downarrow x_{n}\right)$ when $\left(x_{n}\right)_{n}$ is increasing (resp. decreasing) and $x$ is the l.u.b. (resp. g.l.b.) of $\left(x_{n}\right)_{n}$. We will say that $P$ is monotone $\sigma$-complete [7] when every increasing (resp. decreasing) bounded sequence of elements of $P$ has a l.u.b. (resp. g.l.b.).

Formal variable symbols will be written in boldface roman letters $\mathbf{a}, \mathbf{b}$, $\mathbf{x}, \mathbf{y}, \ldots$, while elements of a given structure will be written in math italics $a, b, x, y, \ldots$ Lists of variables or of elements of a structure will often be denoted by vectors $\overrightarrow{\mathbf{x}}, \vec{x}, \ldots$, or $\overrightarrow{\mathbf{x}}$ if $\overrightarrow{\mathbf{x}}=\left(\mathbf{x}_{i}\right)_{i<n}$.

1. Countable bounded atomic compactness. The goal of this section is to recall some classical definitions about (countable or arbitrary) atomic compactness, as well as the version we shall use throughout this paper, bounded (countable or arbitrary) atomic compactness. We refer to [1, $10,15,16]$ for more details about atomic (and equational) compactness. We will here be concerned only with models of the language $(+, \leq)$, but what follows in this section could often be easily generalized to the case of an arbitrary first-order language; it could also be generalized to the case of any infinite cardinal instead of just $\aleph_{0}$.

Recall that an atomic formula is a formula of the form $\sum_{i<n} p_{i} \mathbf{x}_{i} \leq$ $\sum_{i<n} q_{i} \mathbf{x}_{i}$ or $\sum_{i<n} p_{i} \mathbf{x}_{i}=\sum_{i<n} q_{i} \mathbf{x}_{i}$, where $p_{i}$ and $q_{i}$ are positive integers and $\mathbf{x}_{i}$ are variable symbols. A formula is a well-formed expression built up from atomic formulæ and the logical connectives "and", "or", $\neg, \forall$ and $\exists$.

A structure will be by definition a set $A$ equipped with a binary operation + and a binary relation $\leq$. One defines as usual formulæ with parameters 
from a given structure, and the satisfaction relation $A=\varphi$ is to be read "A satisfies $\varphi$ " [4]. The reduced power * $A$ of $A$ by the Fréchet filter over the integers is the quotient structure of ${ }^{\omega} A$ (equipped with componentwise + and $\leq$ ) by the equivalence modulo the Fréchet filter on $\omega[4]$. We will as usual identify $A$ with its image in ${ }^{*} A$ under the natural embedding.

By analogy with equation systems, we will say that a set of formulæ is a system, then using "solvable" instead of "satisfiable". An atomic system is a system of atomic formulæ. If $A$ is a structure, then we will say that $A$ is atomic compact (resp. countably atomic compact) when for every system (resp. countable system) $\Sigma$ of atomic formulæ of $(+, \leq)$, possibly with parameters from $A$, if $\Sigma$ is finitely solvable in $A$ (i.e. every finite subsystem of $\Sigma$ is solvable in $A$ ), then $\Sigma$ is solvable in $A$. When the formulæ that we consider are equations, we obtain the classical definition of equational compactness $[10,16]$. It is well known that if $A$ is a compact structure (i.e. there exists a compact Hausdorff topology on the underlying set for which $\leq$ is closed and + is continuous), then $A$ is atomic compact. The converse is false in a lot of [most] natural cases (see for example $[5,6,12]$ ).

It is well known [16, Theorem 2.3] that a structure is atomic compact if and only if it is a retract of all its reduced powers. The following proposition is a countable analogue of this result:

1.1. Proposition. Let $A$ be a structure. The following are equivalent:

(i) A is countably atomic compact.

(ii) Let $A_{0}$ and $A_{1}$ be countable structures such that $A_{0} \subseteq A$ and $A_{0} \subseteq$ $A_{1} \subseteq{ }^{*} A_{0}$. Then there exists a "partial retraction" from $A_{1}$ to $A$, i.e. a $(+, \leq)$-homomorphism @ from $A_{1}$ to $A$ such that $\varrho \uparrow_{A_{0}}=\mathrm{id}$.

P r o o f. (i) $\Rightarrow$ (ii). Consider the following countable atomic system $\Sigma$, with parameters from $A_{0}$ and with unknowns $\mathbf{x}_{a}\left(a \in A_{1}\right)$ :

$$
\Sigma: \begin{cases}\mathbf{x}_{a+b}=\mathbf{x}_{a}+\mathbf{x}_{b} & \left(\text { for all } a, b \text { in } A_{1}\right), \\ \mathbf{x}_{a} \leq \mathbf{x}_{b} & \left(\text { for all } a, b \text { in } A_{1} \text { such that } a \leq b\right), \\ \mathbf{x}_{a}=a & \left(\text { for all } a \in A_{0}\right)\end{cases}
$$

It is easy to see that $\Sigma$ is finitely solvable in $A$ (and even in $A_{0}$ ); by assumption, $\Sigma$ is solvable in $A$. Any solution of $\Sigma$ in ${ }^{A_{1}} A$ defines a partial retraction from $A_{1}$ to $A$.

(ii) $\Rightarrow$ (i). Let $\Sigma$ be a countable atomic system with parameters from $A$ and with unknowns $\mathbf{x}_{n}(n \in \omega)$. There exists a countable substructure $A_{0}$ of $A$ such that all parameters in $\Sigma$ are in $A_{0}$ and $\Sigma$ is finitely solvable in $A_{0}$. Hence, $\Sigma$ is solvable in ${ }^{*} A_{0}$ [16, Lemma 2.2], and thus in some countable substructure $A_{1}$ of ${ }^{*} A_{0}$ containing $A_{0}$. By assumption, there exists a partial retraction $\varrho$ from $A_{1}$ to $A$; the image under $\varrho$ of a solution of $\Sigma$ in $A_{1}$ is a solution of $\Sigma$ in $A$. 
Note that the proof of (ii) $\Rightarrow$ (i) above does not work when $\Sigma$ consists of arbitrary positive formulæ, even upon replacing ${ }^{*} A_{0}$ by an ultrapower of $A_{0}$ and requiring $A_{0} \prec A$ and $A_{1} \prec{ }^{*} A_{0}$ (and this is a difference with the case of full atomic compactness): indeed, $\varrho\left[A_{1}\right]$ does not seem a priori to be a pure substructure of $A$.

A nonzero directed ordered group $A$ can never be atomic compact (consider the atomic system $\{a \leq \mathrm{x}$ (for all $a \in A$ )); on the other hand, the author proved [21, Theorem 5.5] that if $A$ is a Dedekind complete $f$-ring, then $A$ is "boundedly algebraically [or atomic] compact" in the language $(+,-, \cdot, \wedge, \vee, \leq)$, i.e. every finitely solvable system of the form

$$
\begin{cases}\left.\varphi_{i} \underset{(J)}{\overrightarrow{\mathbf{x}}}\right) & \left(\text { for all } i \in I ; \text { the } \varphi_{i}\right. \text { 's are atomic formulæ } \\ & \text { of the language }(+,-, \cdot, \wedge, \vee, \leq)), \\ \left|\mathbf{x}_{j}\right| \leq a_{j} & \left(\text { for all } j \in J ; \text { the } a_{j} \text { 's belong to } A\right),\end{cases}
$$

is solvable.

1.2. Definition. Let $A$ be a structure, and let $\Sigma$ be a system with parameters from $A$ and with unknowns $\mathbf{x}_{i}(i \in I)$. Then a family $\left(a_{i}, b_{i}\right)_{i \in I}$ of elements of $A \times A$ is bounding for $\Sigma$ when the system

$$
\left\{\begin{array}{l}
\Sigma, \\
a_{i} \leq \mathbf{x}_{i} \leq b_{i}
\end{array} \quad(\text { for all } i \in I),\right.
$$

is finitely solvable. We say that $\Sigma$ is bounding when it admits a bounding family. We say that $A$ is boundedly countably atomic compact when every bounding countable system with parameters from $A$ is solvable in $A$.

For every abelian ordered group $A$, equip $A^{+} \cup\{\infty\}$ with the ordered monoid structure extending $A^{+}$such that $\infty>0$ and $(\forall x)(x+\infty=\infty+x$ $=\infty)$. Then we have the following

1.3. Proposition. Let $A$ be a directed abelian ordered group. Then the following are equivalent:

(i) A is boundedly countably atomic compact.

(ii) $A^{+} \cup\{\infty\}$ is countably atomic compact.

(iii) $A^{+} \cup\{\infty\}$ is countably equationally compact.

Proof. (ii) $\Rightarrow$ (i) is easy. Now assume (i). Put $E=A^{+} \cup\{\infty\}$. Let $C$ be a countable submonoid of $E$, and let $C^{\prime}$ be a countable monoid such that $C \subseteq C^{\prime} \subseteq{ }^{*} C$. Put $I=\left\{\left[x_{n}\right]_{n} \in C^{\prime}:\left(\exists x \in A^{+}\right)(\forall n \in \omega)\left(x_{n} \leq x\right)\right\}$ (the set of all "bounded" elements of $\left.C^{\prime}\right)$. Then we see as in the proof of Proposition $1.1(\mathrm{i}) \Rightarrow$ (ii) (the corresponding system $\Sigma$ is bounding) that by bounded countable atomic compactness of $A$, there exists a partial retraction $\varrho$ from $I$ to $A^{+}$. Thus, the extension $\varrho^{\prime}$ of $\varrho$ to $E$ defined by $\varrho^{\prime}(x)=\infty$ if $x \in C^{\prime} \backslash I$ is a partial retraction from $C^{\prime}$ to $E$ (see also [21, Corollary 5.6] for a similar 
argument). Finally, (ii) $\Leftrightarrow$ (iii) is easy, by observing that in $A^{+} \cup\{\infty\}, x \leq y$ is equivalent to $(\exists z)(x+z=y)$.

Note that the analogue of Proposition 1.3 fails for richer languages, as e.g. for the language $(+, \wedge, \leq)$ and $A=\mathbb{R} \times \mathbb{R}$, with $x \wedge y=\min (x, y)$.

2. Special sentences. Case of monotone $\sigma$-complete groups. Recall [7] that an abelian ordered group $A$ has the interpolation property (resp. the countable interpolation property) when for all finite (resp. countable) nonempty subsets $X$ and $Y$ of $A$ such that $X \leq Y$, there exists $z$ in $A$ such that $X \leq z \leq Y$. Moreover, $A$ is unperforated when for all $m \in \mathbb{N} \backslash\{0\}, A$ satisfies $(\forall \mathbf{x})(m \mathbf{x} \geq 0 \Rightarrow \mathbf{x} \geq 0)$. A dimension group is a directed, abelian, unperforated ordered group having the interpolation property, and a $d i$ mension cone is the positive cone of a dimension group. Recall [7, Theorem $16.10]$ that every abelian monotone $\sigma$-complete group with the (finite) interpolation property is Archimedean and has the countable interpolation property.

The following definition of special sentences matches the one in [3, p. 181] in many aspects. The terminology "linear formula" is chosen by analogy with the already existing term "linear system".

2.1. Definition. A formula of the language $(+, \leq)$ is linear when it is a finite conjunction of atomic formulæ. A special sentence is a formula of the form

$$
(\forall \overrightarrow{\mathbf{x}})[\varphi(\overrightarrow{\mathbf{x}}) \Rightarrow(\exists \overrightarrow{\mathbf{y}}) \psi(\overrightarrow{\mathbf{x}}, \overrightarrow{\mathbf{y}})]
$$

where $\varphi$ and $\psi$ are linear formulæ.

A key fact for this section is the following

2.2. Proposition. There exist computable functions which associate with each linear formula $\varphi \underset{(n)}{(\overrightarrow{\mathbf{x}}})$ a strictly positive integer $N_{\varphi}$ and an $N_{\varphi}$-uple $\left(\vec{m}_{j}\right)_{j<N_{\varphi}}$ of elements of ${ }^{n} N_{\varphi}$ such that for every dimension group $A$,

$$
A^{+} \models(\forall \overrightarrow{\mathbf{x}})\left[\varphi(\overrightarrow{\mathbf{x}}) \Leftrightarrow\left(\exists_{j<N_{\varphi}} \mathbf{y}_{j}\right)\left(\overrightarrow{\mathbf{x}}=\sum_{j<N_{\varphi}} \vec{m}_{j} \mathbf{y}_{j}\right)\right] .
$$

(Thus, taking $A=\mathbb{Z}$, we see that for all $j<N_{\varphi}, \mathbb{N} \models \varphi\left(\vec{m}_{j}\right)$.)

$\operatorname{Proof}$. When $\varphi$ is $s=t$ where $s$ and $t$ are terms, this is [7, Proposition 3.15] (the proof in [7] is obviously effective). When $\varphi$ is $s(\overrightarrow{\mathbf{x}}) \leq t(\overrightarrow{\mathbf{x}})$ where $s$ and $t$ are terms, note that in every dimension cone, the formulæ $\varphi(\overrightarrow{\mathbf{x}})$ and $(\exists \mathbf{y})(s(\overrightarrow{\mathbf{x}})+\mathbf{y}=t(\overrightarrow{\mathbf{x}}))$ are equivalent, and thus it suffices to apply the previous result to the formula $s(\overrightarrow{\mathbf{x}})+\mathbf{y}=t(\overrightarrow{\mathbf{x}})$. Now suppose that the theorem has been proved for conjunctions of $n$ atomic formulæ $(n \in \omega \backslash\{0\})$ and let $\varphi \underset{(n)}{(\overrightarrow{\mathbf{x}}})$ be a conjunction of $n+1$ atomic formulæ. Thus $\varphi$ is $\psi \wedge \theta$, 
where $\psi$ is a conjunction of $n$ atomic formulæ and $\theta$ is atomic. Let $N_{\psi}$ and $\vec{m}_{j}\left(j<N_{\psi}\right)$ be associated with $\psi$ (induction hypothesis). Then, in every dimension cone, $\varphi(\overrightarrow{\mathbf{x}})$ is equivalent to the formula

$$
\left(\exists_{j<N_{\psi}} \mathbf{y}_{j}\right)\left(\overrightarrow{\mathbf{x}}=\sum_{j<N_{\psi}} \vec{m}_{j} \mathbf{y}_{j} \text { and } \theta(\overrightarrow{\mathbf{x}})\right),
$$

so it suffices to apply the case $n=1$ to the atomic formula $\theta\left(\sum_{j<N_{\psi}} \vec{m}_{j} \mathbf{y}_{j}\right)$ and substitute back into $\overrightarrow{\mathbf{x}}$ the expressions found for the $\mathbf{y}_{j}$ 's.

In the context above, $\overrightarrow{\mathbf{x}}=\sum_{j<N_{\varphi}} \vec{m}_{j} \mathbf{y}_{j}$ will be called the general solution of $\varphi(\overrightarrow{\mathbf{x}})$ in dimension cones.

2.3. Corollary. Consider a special sentence $\theta$ :

$$
(\forall \underset{(m)}{\overrightarrow{\mathbf{x}}})(\varphi(\overrightarrow{\mathbf{x}}) \Rightarrow(\exists \underset{(n)}{\overrightarrow{\mathbf{y}}}) \psi(\overrightarrow{\mathbf{x}}, \overrightarrow{\mathbf{y}})),
$$

where $\varphi$ and $\psi$ are linear formulce. Then the following are equivalent:

(i) Every dimension cone satisfies $\theta$.

(ii) $\mathbb{N}$ satisfies $\theta$.

(iii) For all $\vec{x}$ in ${ }^{m} N_{\varphi}$, there exists $\vec{y}$ in ${ }^{n} \mathbb{N}$ such that $\mathbb{N}=\psi(\vec{x}, \vec{y})$.

Proof. (i) $\Rightarrow$ (ii) and (ii) $\Rightarrow$ (iii) are trivial. Now assume (iii). Let $A$ be a dimension group; we prove that $A^{+} \models \theta$. So let $\vec{x}$ in ${ }^{m} A^{+}$be such that $A^{+} \models \varphi(\vec{x})$. Let $N_{\varphi}$ and $\vec{m}_{j}\left(j<N_{\varphi}\right)$ be associated with $\varphi$ as in Proposition 2.2. By Proposition 2.2, there are $t_{j}\left(j<N_{\varphi}\right)$ in $A^{+}$such that $\vec{x}=\sum_{j<N_{\varphi}} \vec{m}_{j} t_{j}$. Since $\vec{m}_{j} \in{ }^{m} N_{\varphi}$ for all $j$ and $\mathbb{N} \models \varphi\left(\vec{m}_{j}\right)$, there are $\vec{n}_{j}\left(j<N_{\varphi}\right)$ in $\mathbb{N}_{\mathbb{N}}$ such that for all $j, \mathbb{N}=\psi\left(\vec{m}_{j}, \vec{n}_{j}\right)$. Put $\vec{y}=\sum_{j<N_{\varphi}} \vec{n}_{j} t_{j}$. Then $\vec{y} \in{ }^{n} A^{+}$and $A^{+}=\psi(\vec{x}, \vec{y})$.

2.4. Corollary. Let $\varphi(\mathbf{x}, \underset{(m)}{\overrightarrow{\mathbf{x}}} \underset{(n)}{\overrightarrow{\mathbf{a}}})$ be a linear formula. Then for every dimension group $A$ and all $\vec{a}$ in ${ }^{n} A^{+}$, the "projection"

$$
D=\left\{x \in A^{+}: A^{+} \models(\exists \overrightarrow{\mathbf{x}}) \varphi(x, \overrightarrow{\mathbf{x}}, \vec{a})\right\}
$$

is both filtered and directed.

Proof. The statement that $D$ is always directed means that the following sentence $\theta$ is satisfied by all dimension cones:

$$
\begin{aligned}
& (\forall \mathbf{x}, \mathbf{y}, \overrightarrow{\mathbf{x}}, \overrightarrow{\mathbf{y}}, \overrightarrow{\mathbf{a}})[(\varphi(\mathbf{x}, \overrightarrow{\mathbf{x}}, \overrightarrow{\mathbf{a}}) \text { and } \varphi(\mathbf{y}, \overrightarrow{\mathbf{y}}, \overrightarrow{\mathbf{a}})) \Rightarrow \\
& \qquad(\exists \mathbf{z}, \overrightarrow{\mathbf{z}})(\mathbf{x} \leq \mathbf{z} \text { and } \mathbf{y} \leq \mathbf{z} \text { and } \varphi(\mathbf{z}, \overrightarrow{\mathbf{z}}, \overrightarrow{\mathbf{a}}))] .
\end{aligned}
$$

However, $\theta$ is special, thus, by Corollary 2.4, it suffices to show that $\theta$ holds in $\mathbb{N}$. However, since $\mathbb{N}$ is linearly ordered, this is obvious: if e.g. $x \leq y$, then take $z=y$ and $z_{i}=y_{i}$ for all $i$. The proof for " $D$ is filtered" is similar.

The idea underlying the use of Corollary 2.4 for the proof of bounded countable atomic compactness of monotone $\sigma$-complete dimension groups is 
the following: if $S\left(\mathbf{x}_{0}, \mathbf{x}_{1}, \ldots\right)$ is a countable bounding atomic system with parameters from a given monotone $\sigma$-complete dimension group $A$, one constructs, using Corollary 2.4, a bounded increasing sequence of $x_{0}$ 's such that larger and larger finite subsystems of $S\left(x_{0}, \mathbf{x}_{1}, \mathbf{x}_{2}, \ldots\right)$ are finitely solvable. But now, we have to prove that the l.u.b. of such a sequence is in the first projection of $S$ ! Basically, it would be good if with an increasing sequence of $x_{0}$ 's, one could e.g. associate a decreasing, or increasing, sequence of $x_{1}$ 's; however, easy examples show that this is not always possible. Now, thinking "with bounded variation" instead of "increasing" or "decreasing" yields a small trick, summarized in the following proposition:

2.5. Corollary. Let $\varphi(\mathbf{x}, \underset{(m)}{\overrightarrow{\mathbf{x}}}, \underset{(n)}{\overrightarrow{\mathbf{a}}})$ be a linear formula, and let $N=N_{\psi}$, where $\psi$ is the following linear formula:

$$
\varphi(\mathbf{x}, \overrightarrow{\mathbf{x}}, \overrightarrow{\mathbf{a}}) \text { and } \varphi(\mathbf{y}, \overrightarrow{\mathbf{y}}, \overrightarrow{\mathbf{a}}) \text { and } \mathbf{x} \leq \mathbf{y} .
$$

Let $A$ be a dimension group, and let $\vec{a} \in{ }^{n} A^{+}$. Put

$$
\begin{aligned}
D & =\left\{x \in A^{+}: A^{+}=(\exists \overrightarrow{\mathbf{x}}) \varphi(x, \overrightarrow{\mathbf{x}}, \vec{a})\right\}, \\
S & =\left\{(x, \vec{x}) \in A^{+} \times{ }^{n} A^{+}: A^{+}=\varphi(x, \vec{x}, \vec{a})\right\} .
\end{aligned}
$$

Then for all $(x, \vec{x}) \in S$ and all $y \in D$ such that $x \leq y$, there exists $\vec{y} \in{ }^{n} A^{+}$ such that $(y, \vec{y}) \in S$ and for all $i<m, N x+x_{i} \leq N y+y_{i}$.

Proof. It suffices to prove that the following sentence $\theta$ holds in every dimension cone:

$$
\begin{aligned}
& (\forall \mathbf{x}, \mathbf{y}, \overrightarrow{\mathbf{x}}, \overrightarrow{\mathbf{y}}, \overrightarrow{\mathbf{a}})[(\varphi(\mathbf{x}, \overrightarrow{\mathbf{x}}, \overrightarrow{\mathbf{a}}) \text { and } \varphi(\mathbf{y}, \overrightarrow{\mathbf{y}}, \overrightarrow{\mathbf{a}}) \text { and } \mathbf{x} \leq \mathbf{y}) \Rightarrow \\
& \left.(\exists \overrightarrow{\mathbf{z}})\left(\bigwedge_{i<m}\left(N \mathbf{x}+\mathbf{x}_{i} \leq N \mathbf{y}+\mathbf{z}_{i}\right) \text { and } \varphi(\mathbf{y}, \overrightarrow{\mathbf{z}}, \overrightarrow{\mathbf{a}})\right)\right] .
\end{aligned}
$$

However, $\theta$ is special, thus, by Corollary 2.4, it suffices to show that it holds in $\mathbb{N}$ for $x, y$ and components of $\vec{a}, \vec{x}, \vec{y}$ strictly less than $N$. If $x=y$ then just take $z_{i}=x_{i}$ (for all $i<m$ ). So suppose $x<y$; take $z_{i}=y_{i}$ (for all $i<m)$. We verify that $N x+x_{i} \leq N y+y_{i}$, i.e. $x_{i}-y_{i} \leq N(y-x)$. However, this is the case, since $x_{i}-y_{i} \leq x_{i}<N \leq N(y-x)$.

2.6. Lemma. Let $\varphi(\underset{(m)}{\underset{(}{\overrightarrow{\mathbf{x}}}, \underset{(n)}{\overrightarrow{\mathbf{a}}})}$ be a linear formula, let $A$ be a monotone $\sigma$-complete dimension group, let $\vec{a} \in{ }^{n} A$, and let $\vec{x}, \vec{x}_{k}(k \in \omega)$ be elements of $A$ such that $\vec{x}=\bigvee_{k} \uparrow \vec{x}_{k}\left(\right.$ resp. $\left.\vec{x}=\bigwedge_{k} \downarrow \vec{x}_{k}\right)$ and for all $k, A=\varphi\left(\vec{x}_{k}, \vec{a}\right)$. Then $A=\varphi(\vec{x}, \vec{a})$.

Proof. $\varphi(\overrightarrow{\mathbf{x}}, \vec{a})$ is equivalent to a conjunction of formulæ of the form $\sum_{i<m} p_{i} \mathbf{x}_{i}+a \leq \sum_{i<m} q_{i} \mathbf{x}_{i}+b\left(p_{i}, q_{i} \in \mathbb{N}\right.$ and $\left.a, b \in A^{+}\right)$. Since addition is distributive on countable $\bigvee \uparrow($ resp. $\bigwedge \downarrow$ ), the conclusion follows immediately. 
2.7. Proposition. Let $\varphi(\mathbf{x}, \underset{(m)}{\overrightarrow{\mathbf{x}}}, \underset{(n)}{\overrightarrow{\mathbf{a}}})$ be a linear formula, let $A$ be a monotone $\sigma$-complete dimension group, and let $\vec{a} \in{ }^{n} A$ and $a \in A^{+}$. Then

$$
D_{a}=\left\{x \in A^{+}: A=\left(\exists_{i<m} \mathbf{x}_{i} \leq a\right) \varphi(x, \overrightarrow{\mathbf{x}}, \vec{a})\right\}
$$

is closed under countable $\bigvee \uparrow$ and $\bigwedge \downarrow$.

Proof. We prove the result for countable $\bigvee \uparrow$; the proof for countable $\bigwedge \downarrow$ is similar. So let $\left(x^{k}\right)_{k \in \omega}$ be a countable increasing bounded sequence of elements of $D_{a}$, and let $x=\bigvee_{k} \uparrow x^{k}$. We prove that $x \in D_{a}$. Let $N$ be the integer associated as in Corollary 2.5 with the formula $[\varphi(\mathbf{x}, \overrightarrow{\mathbf{x}}, \overrightarrow{\mathbf{a}})$ and $\left.\mathbb{M}_{i<m}\left(\mathbf{x}_{i} \leq \mathbf{a}\right)\right]$. For all $k \in \omega$, we construct inductively $\vec{x}^{k}=\left(x_{i}^{k}\right)_{i<m}$ $\left(0 \leq x_{i}^{k} \leq a\right)$ in the following way.

- Since $x^{0} \in D_{a}$, there exists $\vec{x}^{0}$ in ${ }^{m}[0, a]$ such that $A=\varphi\left(x^{0}, \vec{x}^{0}, \vec{a}\right)$.

- Suppose that $\vec{x}^{k}$ has been constructed (with $0 \leq x_{i}^{k} \leq a$ ) and $A \models$ $\varphi\left(x^{k}, \vec{x}^{k}, \vec{a}\right)$. Since $x^{k} \leq x^{k+1}$ and both belong to $D_{a}$, there exists, by Corollary $2.5, \vec{x}^{k+1}$ in ${ }^{m}[0, \vec{a}]$ such that $A=\varphi\left(x^{k+1}, \vec{x}^{k+1}, \vec{a}\right)$ and for all $i<m$, $N x^{k}+x_{i}^{k} \leq N x^{k+1}+x_{i}^{k+1}$.

Thus for all $i<m$, the sequence $\left(y_{i}^{k}\right)_{k}$, where $y_{i}^{k}=N x^{k}+x_{i}^{k}$, is increasing. But it is bounded above (by $N x+a$ ), thus it has a l.u.b., say $y_{i}$. Let $\psi(\mathbf{z}, \underset{(m)}{\overrightarrow{\mathbf{z}}}, \overrightarrow{\mathbf{a}})$ be the linear formula $\varphi(\mathbf{z}, \overrightarrow{\mathbf{z}}-N \mathbf{z}, \overrightarrow{\mathbf{a}})$. Then for all $k$, we have $A=\psi\left(x^{k}, \vec{y}^{k}, \vec{a}\right)$, whence, by Lemma $2.6, A=\psi(x, \vec{y}, \vec{a})$, whence $A=\varphi(x, \vec{x}, \vec{a})$, where $\vec{x}=\vec{y}-N x$. Finally, for all $i<m$, we have $0 \leq x_{i}^{k} \leq a$, which can be written $N x^{k} \leq y_{i}^{k} \leq a+N x^{k}$; taking l.u.b. in $k$ of all members yields $N x \leq y_{i} \leq a+N x$, i.e. $0 \leq x_{i} \leq a$. Hence, $x \in D_{a}$.

Now we can prove the decisive lemma:

2.8. LeMmA. Let $A$ be a monotone $\sigma$-complete dimension group, and let $S\left(\mathbf{x}_{0}, \mathbf{x}_{1}, \mathbf{x}_{2}, \ldots\right)$ be a bounding atomic system with parameters from $A$ and with a bounding sequence of the form $\left(0, a_{n}\right)_{n \in \omega}$. Then there exists $x$ in $\left[0, a_{0}\right]$ such that $S\left(x, \mathbf{x}_{1}, \mathbf{x}_{2}, \ldots\right)$ is a bounding system with bounding family $\left(0, a_{n}\right)_{n \in \omega \backslash\{0\}}$ (so that in particular, it is finitely solvable).

Pr o of. Write $S$ as a countable increasing sequence $S=\bigcup_{n \in \omega} S_{n}$ of finite subsystems. Note that each $S_{n}$ can be naturally identified with a linear formula with parameters from $A$. Consider the strict well-ordering $\prec$ on $\omega \times \omega$ defined by $(i, j) \prec\left(i^{\prime}, j^{\prime}\right)$ if and only if $i+j<i^{\prime}+j^{\prime}$ or $\left(i+j=i^{\prime}+j^{\prime}\right.$ and $\left.j<j^{\prime}\right)$. Construct by $\prec$-induction $x_{i}^{j}(i, j \in \omega)$ in the following way. Suppose that all $x_{i^{\prime}}^{j^{\prime}}$ are constructed for $\left(i^{\prime}, j^{\prime}\right) \prec(i, j)$ in such a way that for all these $\left(i^{\prime}, j^{\prime}\right), x_{i^{\prime}}^{j^{\prime}} \in\left[0, a_{0}\right]$ and $S_{i^{\prime}}\left(x_{i^{\prime}}^{j^{\prime}}, \mathbf{x}_{1}, \mathbf{x}_{2}, \ldots\right)$ is solvable in $\prod_{l \geq 1}\left[0, a_{l}\right]$. Put $n=i+j$. If $j=0$, then $i=n$ and there is $x_{n}^{0}$ in $\left[0, a_{0}\right]$ such that $S_{n}\left(x_{n}^{0}, \mathbf{x}_{1}, \mathbf{x}_{2}, \ldots\right)$ is solvable in $\prod_{l \geq 1}\left[0, a_{l}\right]$. If $j>0$, then by Corollary 2.4 
there exists $x_{i}^{j} \leq x_{i+1}^{j-1}, x_{i}^{j-1}$ in $\left[0, a_{0}\right]$ such that $S_{i}\left(x_{i}^{j}, \mathbf{x}_{1}, \mathbf{x}_{2}, \ldots\right)$ is solvable in $\prod_{l \geq 1}\left[0, a_{l}\right]$. The picture is as follows:

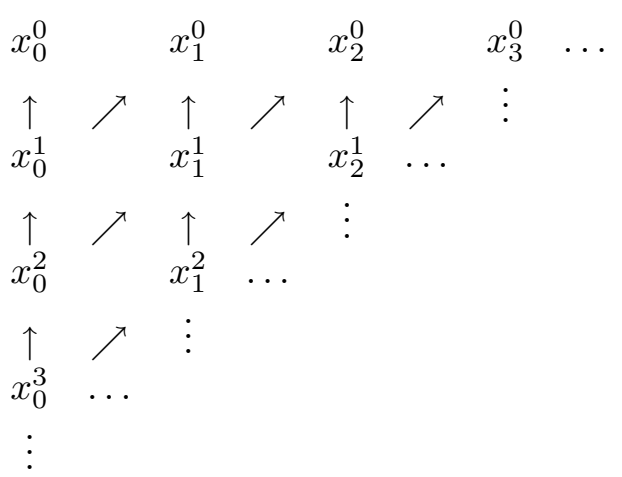

In this diagram, an arrow from $x_{i}^{j}$ to $x_{i^{\prime}}^{j^{\prime}}$ means that $x_{i}^{j} \leq x_{i^{\prime}}^{j^{\prime}}$. Now, for all $m$ in $\omega$, put $x_{m}=\bigwedge_{k} \downarrow x_{m}^{k}$. By Proposition $2.7, S_{m}\left(x_{m}, \mathbf{x}_{0}, \mathbf{x}_{1}, \ldots\right)$ is solvable in $\prod_{l \geq 1}\left[0, a_{l}\right]$; thus $S_{m}\left(x_{n}, \mathbf{x}_{0}, \mathbf{x}_{1}, \ldots\right)$ is solvable in $\prod_{l \geq 1}\left[0, a_{l}\right]$ for all $n \geq m$. Furthermore, it results from the construction that $\left(x_{n}\right)_{n}$ is increasing; so put $x=\bigvee_{n} \uparrow x_{n}$. Thus $x \in\left[0, a_{0}\right]$, and by Proposition 2.7 again, $S_{m}\left(x, \mathbf{x}_{0}, \mathbf{x}_{1}, \ldots\right)$ is solvable in $\prod_{l \geq 1}\left[0, a_{l}\right]$ for all $m \in \omega$. Thus $x$ is as required.

Now we can give the main theorem of this section.

2.9. TheOREM. Every monotone $\sigma$-complete dimension group is boundedly countably atomic compact. Equivalently, for every monotone $\sigma$-complete dimension group $A,\left(A^{+} \cup\{\infty\},+, \leq\right)$ is countably equationally (atomic) compact.

Proof. Let $A$ be a monotone $\sigma$-complete dimension group, and let $S\left(\mathbf{x}_{0}, \mathbf{x}_{1}, \mathbf{x}_{2}, \ldots\right)$ be a countable bounding system with parameters from $A$. We show that $S$ admits a solution in $A$. By appropriate translations of the variables, we may assume without loss of generality that $S$ admits a bounding sequence of the form $\left(0, a_{n}\right)_{n}$. Now, using Lemma 2.8 , it is easy to construct inductively a sequence $\left(x_{n}\right)_{n}$ in $\prod_{n}\left[0, a_{n}\right]$ such that for all $n$, $S\left(x_{0}, \ldots, x_{n}, \mathbf{x}_{n+1}, \mathbf{x}_{n+2}, \ldots\right)$ admits $\left(0, a_{l}\right)_{l>n}$ as a bounding family (thus is finitely solvable). Since every subsystem of $S$ involves only finitely many unknowns, $\left(x_{n}\right)_{n}$ is a solution of $S$ in $\prod_{n}\left[0, a_{n}\right]$.

Another fact is that in Proposition 2.7, the restriction that $0 \leq \mathbf{x}_{i} \leq a$ may be removed as far as formulæ of the language $(+, \leq)$ are concerned (see the following counterexample 2.12 for a richer language).

2.10. Lemma. Let $\varphi(\underset{(m)}{\overrightarrow{\mathbf{x}}}, \underset{(n)}{\overrightarrow{\mathbf{a}}})$ be a linear formula, let $A$ be a monotone $\sigma$-complete dimension group, and let $\vec{a} \in{ }^{n} A^{+}$. Put $a=N_{\varphi} \sum_{j} a_{j}$. Then 


$$
A^{+}=\left[(\exists \overrightarrow{\mathbf{x}}) \varphi(\overrightarrow{\mathbf{x}}, \vec{a}) \Rightarrow(\exists \overrightarrow{\mathbf{x}})\left(\bigwedge_{i<m}\left(0 \leq \mathbf{x}_{i} \leq a\right) \text { and } \varphi(\overrightarrow{\mathbf{x}}, \vec{a})\right)\right] .
$$

Proof. Put $N=N_{\varphi}$. Let $\theta$ be the following statement:

$$
(\forall \overrightarrow{\mathbf{x}}, \overrightarrow{\mathbf{a}})\left[\varphi(\overrightarrow{\mathbf{x}}, \overrightarrow{\mathbf{a}}) \Rightarrow(\exists \overrightarrow{\mathbf{y}}) \bigwedge_{i<m}\left(\left(0 \leq \mathbf{y}_{i} \leq N \sum_{j<n} \mathbf{a}_{j}\right) \text { and } \varphi(\overrightarrow{\mathbf{y}}, \overrightarrow{\mathbf{a}})\right)\right] .
$$

Since $\theta$ is a special sentence, it suffices to prove that $\theta$ is satisfied by $\mathbb{N}$, and furthermore, it suffices to verify $\theta$ for $x_{i}, a_{j}<N$ (Corollary 2.3). So suppose that $x_{i}, a_{j}<N$ for all $i<m, j<n$ and that $\mathbb{N}$ satisfies $\varphi(\vec{x}, \vec{a})$. If $a_{j}=0$ for all $j$, put $y_{i}=0$ for all $i$; otherwise, take $y_{i}=x_{i}$ for all $i$. Then in both cases, $0 \leq y_{i} \leq N \sum_{j} a_{j}$ and $\varphi(\vec{y}, \vec{a})$.

2.11. Theorem. Let $\varphi(\mathbf{x}, \underset{(m)}{\overrightarrow{\mathbf{x}}} \underset{(n)}{\overrightarrow{\mathbf{a}}})$ be a linear formula, let $A$ be a monotone $\sigma$-complete dimension group, and let $\vec{a} \in{ }^{n} A$. Then

$$
D=\{x \in A: A=(\exists \overrightarrow{\mathbf{x}}) \varphi(x, \overrightarrow{\mathbf{x}}, \vec{a})\}
$$

is closed under countable $\bigvee \uparrow$ and $\bigwedge \downarrow$.

Proof. We prove for example that $D$ is closed under countable $\bigvee \uparrow$; the proof for $\Lambda \downarrow$ is similar. So let $x$ in $A$ and $x_{k}(k \in \omega)$ in $D$ be such that $x=\bigvee_{k} \uparrow x_{k}$. We prove that $x \in D$. Since $A$ is directed, one can assume without loss of generality that $x_{0} \geq 0$ and $a_{j} \geq 0$ for all $j<n$, whence $x \geq 0$ and $x_{k} \geq 0$ for all $k$. Furthermore, for all $y$ in $A^{+}$, we have

$$
y \in D \Leftrightarrow A=(\exists \overrightarrow{\mathbf{z}}, \overrightarrow{\mathbf{t}} \geq 0) \varphi(y, \overrightarrow{\mathbf{z}}-\overrightarrow{\mathbf{t}}, \vec{a}) .
$$

Thus there exists a linear formula $\psi(\mathbf{x}, \underset{(2 m)}{\overrightarrow{\mathbf{x}}}, \underset{(n)}{\overrightarrow{\mathbf{a}}})$ such that $D \cap A^{+}=$ $\left\{y \in A^{+}: A^{+}=\psi(y, \overrightarrow{\mathbf{x}}, \vec{a})\right\}$. By Lemma 2.10, if $N=N_{\psi}$, we also have

$$
D=\left\{y \in A^{+}: A^{+}=\left(\exists_{i<2 m} \mathbf{x}_{i} \leq N\left(y+\sum_{j<n} a_{j}\right)\right) \psi(y, \overrightarrow{\mathbf{x}}, \vec{a})\right\} .
$$

Thus for all $k$ in $\omega, x_{k} \in D_{a}$, where $a=N\left(x+\sum_{j<n} a_{j}\right)$ and

$$
D_{a}=\left\{y \in A^{+}: A^{+} \models\left(\exists_{i<2 m} \mathbf{x}_{i} \leq a\right) \psi(y, \overrightarrow{\mathbf{x}}, \vec{a})\right\} .
$$

By Proposition 2.7, $x \in D_{a}$, whence $x \in D$.

2.12. ExAmple. The analogue of Theorem 2.11 may fail for richer languages. Let $\mathcal{L}=(+, \cdot, \leq)$, where + and $\cdot$ are binary operation symbols and $\leq$ is a binary relation symbol. Consider the natural realization of $\mathbb{R}$ as a model of $\mathcal{L}$. Then

$$
D=\{x \in \mathbb{R}: \mathbb{R}=(\exists \mathbf{y})(x \cdot \mathbf{y}=1)\}
$$

is not closed under countable increasing suprema or infima. 
2.13. Question. Let $A$ be a monotone $\sigma$-complete dimension group. Is the structure $(A \cup\{\infty\},+, \leq)$ countably positive compact? Positive formulæ are as usual those which are built up only from the connectors "and", "or", $\exists, \forall$ (no negation). The answer to this question is affirmative for Dedekind complete $\ell$-groups by [21, Corollary 5.6] (atomic compactness implies positive compactness [16], but observe that the proof does not apply to the countable case).

2.14. Question. Is there a "compactness-like" statement which characterizes monotone $\sigma$-complete dimension groups? See [21, Proposition 5.8] for a related statement.

2.15. Question. It is easy to verify that any boundedly countably atomic compact dimension group has the countable interpolation property. Is the converse true (at least for Archimedean groups)?

3. Consequences of bounded countable atomic compactness. We choose in this chapter two kinds of problems that can be formulated in terms of equations-and-inequalities systems. The first kind has been studied in [13] and it deals with extensions of semigroup-valued charges. By definition, if $\mathcal{A}$ is a Boolean algebra and $E$ is a preordered commutative monoid, then $\mu: \mathcal{A} \rightarrow E$ is a charge when $\mu(0)=0$ and whenever $x$, $y$ are disjoint elements of $\mathcal{A}$, then $\mu(x \vee y)=\mu(x)+\mu(y)$. If $\mathcal{A}$ and $\mathcal{B}$ are Boolean subalgebras of some Boolean algebra $\mathcal{C}$ and $\mu: \mathcal{A} \rightarrow E$ and $\nu: \mathcal{B} \rightarrow E$ are charges, then $\mu$ and $\nu$ are said to be consistent when for all $x \in \mathcal{A}$ and $y \in \mathcal{B}, x \leq y$ (resp. $x \geq y, x=y$ ) implies that $\mu(x) \leq \nu(y)$ (resp. $\mu(x) \geq \nu(y), \mu(x)=\nu(y))$. This does not imply in general that $\mu$ and $\nu$ admit a common extension to an $E$-valued charge on $\mathcal{C}$, but there are important cases where it does. In [13, Theorem 3.2], the class of those positively preordered commutative monoids $E$ such that any two consistent $E$-valued charges with finite domains admit a common extension is characterized by a very simple finite set of conditions. Among these conditions is "minimality" of the preordering, i.e. for all $x, y$ in $E, x \leq y$ if and only if there exists $z$ such that $x+z=y$. Structures with this "grid property" are for example all abelian groups (with the coarse preordering), but also $A^{+} \cup\{\infty\}$, where $A$ is any interpolation group. Now, in the case where $\left(A^{+} \cup\{\infty\},+, \leq\right)$ is atomic compact, the extension property is satisfied in the general case, i.e. any two consistent $A^{+} \cup\{\infty\}$-valued charges admit a common extension; this includes the case where $A$ is a Dedekind complete $\ell$-group [21, Corollaries 5.6, 5.7]. Now, when $A$ is just monotone $\sigma$-complete, this may fail, but the argument above is easily seen to work in the case where only countable systems are involved. Thus we get the following 
3.1. Proposition. Let $A$ be a boundedly countably atomic compact interpolation group. Then $A^{+} \cup\{\infty\}$ has the 2-charge extension property for countable charges. This holds in particular when $A$ is a monotone $\sigma$ complete dimension group.

Another field of application of the results of the previous chapter lies in the generalization of the results of [7, pp. 37-43] about relatively bounded homomorphisms. First recall

3.2. Definition. Let $X$ and $Y$ be ordered sets. A map $f: X \rightarrow Y$ is relatively bounded when for every bounded subset $W$ of $X, f[W]$ is bounded in $Y$.

Then we can obtain the following analogue of [7, Proposition 2.25].

3.3. Proposition. Let $A$ be a countable dimension group, and let $E$ be a boundedly countably atomic compact dimension group, and let $f \in$ $\operatorname{Hom}_{\mathbb{Z}}(A, E)$. Then $f$ is relatively bounded if and only if there are positive homomorphisms $g$ and $h$ such that $f=g-h$. This holds in particular when $A$ is a countable dimension group and $E$ is a monotone $\sigma$-complete dimension group.

Proof. If $f=g-h$ with positive $g, h$, then it is immediate that $f$ is relatively bounded. So suppose now that $f$ is relatively bounded. For all $a$ in $A^{+}$, there exists $\varphi(a)$ in $E^{+}$such that $(\forall x \in[0, a])(0 \leq f(x) \leq \varphi(a))$. We need

Claim. Let $G$ be a dimension group, and let $m, n$ in $\omega \backslash\{0\}, a_{i}(i<m)$ in $G, \vec{p}_{j}=\left(p_{i j}\right)_{i<m}$ in ${ }^{m_{\mathbb{N}}}($ for all $j<n)$, and $b_{j}(j<n)$ in $G^{+}$be such that for all $I \subseteq m$ and all $j<n, \sum_{i \in I} p_{i j} a_{i} \leq b_{j}$. Then the following system with unknowns $\mathbf{x}_{i}(i<m)$ is solvable:

$$
\begin{cases}0, a_{i} \leq \mathbf{x}_{i} & (\text { for all } i<m), \\ \sum_{i<m} p_{i j} \mathbf{x}_{i} \leq b_{j} & (\text { for all } j<n) .\end{cases}
$$

Pro of of claim. By Corollary 2.3, it is sufficient to prove the claim in the case where $G=\mathbb{Z}$. But in this case, put $x_{i}=\max \left(a_{i}, 0\right)$. Then for all $i<m$, we have $0, a_{i} \leq x_{i}$. Furthermore, let $j<n$. Put $I=\left\{i<m: a_{i} \geq 0\right\}$. Then $\sum_{i<m} p_{i j} x_{i}=\sum_{i \in I} p_{i j} a_{i} \leq b_{j}$. Hence, $\left(x_{i}\right)_{i<m}$ is a solution of the system.

Now, consider the following atomic system $S$, with unknowns $\mathbf{x}_{a}, a \in A^{+}$:

$$
S: \begin{cases}0, f(a) \leq \mathbf{x}_{a} \leq \varphi(a) & \left(\text { for all } a \in A^{+}\right) \\ \mathbf{x}_{a+b}=\mathbf{x}_{a}+\mathbf{x}_{b} & \left(\text { for all } a, b \text { in } A^{+}\right) .\end{cases}
$$

We prove that $S$ is finitely solvable. So let $P$ be a finite subset of $A^{+}$, and consider the following finite system $S_{P}$ :

$$
S_{P}: \begin{cases}0, f(a) \leq \mathbf{x}_{a} \leq \varphi(a) & (\text { for all } a \in P) \\ \mathbf{x}_{a+b}=\mathbf{x}_{a}+\mathbf{x}_{b} & (\text { for all } a, b \text { in } P) .\end{cases}
$$


Let $S_{P}^{\prime}$ be the system $\left\{\mathbf{x}_{a+b}=\mathbf{x}_{a}+\mathbf{x}_{b}\right.$ (for all $a, b$ in $P$ ). It is a finite equation system with unknowns $\mathbf{x}_{a}(a \in Q)$, where $Q=P \cup(P+P)$. By Proposition 2.2, $S_{P}^{\prime}$ admits a general solution $\mathbf{x}_{a}=\sum_{k<N} n_{a k} \mathbf{t}_{k}$ in all dimension cones. In particular, for all $a, b$ in $P$ and all $k<N$, we have $n_{a+b, k}=n_{a k}+n_{b k}$. Furthermore, since $\left(\mathbf{x}_{a} \mapsto a\right)$ is a solution of $S_{P}^{\prime}$ in $A^{+}$and $A$ is a dimension group, there are $b_{k}(k<N)$ in $A^{+}$such that $(\forall a \in Q)\left(a=\sum_{k<N} n_{a k} b_{k}\right)$. Now consider the following system $R$ with unknowns $\mathbf{y}_{k}(k<N)$ :

$$
R: \begin{cases}0, f\left(b_{k}\right) \leq \mathbf{y}_{k} & (\text { for all } k<N), \\ \sum_{k<N} n_{a k} \mathbf{y}_{k} \leq \varphi(a) & (\text { for all } a \in Q) .\end{cases}
$$

Let $a \in Q, I \subseteq N$. We have

$$
\sum_{k \in I} n_{a k} f\left(b_{k}\right)=f(\underbrace{\sum_{k \in I} n_{a k} b_{k}}_{\in[0, a]}) \leq \varphi(a),
$$

so that the condition of the claim is satisfied. Therefore, $R$ admits a solution, say $\left(y_{k}\right)_{k<N}$. For all $a \in Q$, put $x_{a}=\sum_{k<N} n_{a k} y_{k}$. Then for all $a \in P$, $x_{a} \geq 0, x_{a} \geq \sum_{k<N} n_{a k} f\left(b_{k}\right)=f(a)$, and $x_{a} \leq \varphi(a)$. Furthermore, for all $a$, $b$ in $P$, we have $(\forall k<N)\left(n_{a+b, k}=n_{a k}+n_{b k}\right)$, whence $x_{a+b}=x_{a}+x_{b}$. Thus $\left(x_{a}\right)_{a \in Q}$ is a solution of $S_{P}$. Therefore, $S$ is finitely solvable, hence bounding with bounding family $(0, \varphi(a))_{a \in A^{+}}$. By assumption, $S$ admits a solution, say $\left(x_{a}\right)_{a \in A^{+}}$. The map $a \mapsto x_{a}$ extends naturally to a positive homomorphism $g$ from $A$ to $E$, and by assumption, $f \leq g$. Then $g$ and $h=g-f$ satisfy the required conditions. The final conclusion comes from Theorem 2.9.

When $A$ and $E$ are directed abelian ordered groups, equip $\operatorname{Hom}_{\mathbb{Z}}(A, E)$, as in [7], with the partial ordering $\leq^{+}$whose positive cone consists of the positive homomorphisms. The set $\operatorname{Hom}_{\mathbb{Z}}^{*}(A, E)$ of all relatively bounded homomorphisms from $A$ to $E$ is an ideal of $\operatorname{Hom}_{\mathbb{Z}}(A, E)$. The first statement of the following theorem is a Hahn-Banach like theorem for boundedly countably atomic compact dimension groups.

3.4. TheOrem. Let $A$ be a countable dimension group, and let $E$ be a boundedly countably atomic compact dimension group. Then:

(a) Let $X$ and $Y$ be nonempty, countable sets of maps from $A^{+}$to $E$ such that $X \leq Y$ and:

(i) $(\forall f \in X)\left(\forall a, b \in A^{+}\right)(f(a+b) \leq f(a)+f(b))$;

(ii) $(\forall g \in Y)\left(\forall a, b \in A^{+}\right)(g(a+b) \geq g(a)+g(b))$.

Then there exists $h$ in $\operatorname{Hom}_{\mathbb{Z}}(A, E)$ such that

$$
(\forall f \in X)(\forall g \in Y)\left(f \leq h \uparrow_{A^{+}} \leq g\right) .
$$


(b) Suppose that $E$ is monotone $\sigma$-complete. Then $\operatorname{Hom}_{\mathbb{Z}}(A, E)$ is a monotone $\sigma$-complete interpolation group and $\operatorname{Hom}_{\mathbb{Z}}^{*}(A, E)$ is a monotone $\sigma$-complete dimension group.

Proof. (a) Consider the following atomic system $S$, with unknowns $\mathbf{x}_{a}$ $\left(a \in A^{+}\right)$:

$$
S: \begin{cases}f(a) \leq \mathbf{x}_{a} \leq g(a) & \left(\text { for all } f \in X, g \in Y, a \in A^{+}\right), \\ \mathbf{x}_{a+b}=\mathbf{x}_{a}+\mathbf{x}_{b} & \left(\text { for all } a, b \text { in } A^{+}\right) .\end{cases}
$$

We show that $S$ is finitely solvable. So let $X_{0} \subseteq X, Y_{0} \subseteq Y, P \subseteq A^{+}$be finite and nonempty, and consider the following system $S_{0}$ :

$$
S_{0}: \begin{cases}f(a) \leq \mathbf{x}_{a} \leq g(a) & \left(\text { for all } f \in X_{0}, g \in Y_{0}, a \in P\right), \\ \mathbf{x}_{a+b}=\mathbf{x}_{a}+\mathbf{x}_{b} & (\text { for all } a, b \text { in } P) .\end{cases}
$$

We prove that $S_{0}$ admits a solution. Consider first the following system $S_{1}$ :

$$
S_{1}:\left\{\mathbf{x}_{a+b}=\mathbf{x}_{a}+\mathbf{x}_{b} \quad(\text { for all } a, b \text { in } P) .\right.
$$

Let $Q=P \cup(P+P)$, and let $\mathbf{x}_{a}=\sum_{k<N} n_{a k} \mathbf{t}_{k}$ be the general solution of $S_{1}$ in dimension cones; thus for all $a, b$ in $P$ and $k<N$, we have $n_{a+b, k}=$ $n_{a k}+n_{b k}$. Furthermore, $\left(\mathbf{x}_{a} \mapsto a\right)$ is a solution of $S_{1}$ in $A^{+}$; therefore, there are $b_{k}(k<N)$ in $A^{+}$such that for all $a \in Q$, we have $a=\sum_{k<N} n_{a k} b_{k}$. Now, since $E$ satisfies the interpolation property, there are $c_{k}(k<N)$ in $E$ such that for all $f \in X_{0}, g \in Y_{0}$ and $k<N$, we have $f\left(b_{k}\right) \leq c_{k} \leq g\left(b_{k}\right)$. For all $a \in Q$, let $x_{a}=\sum_{k<N} n_{a k} c_{k}$. We check that $\left(x_{a}\right)_{a \in Q}$ is a solution of $S_{0}$.

- Let $f \in X_{0}, g \in Y_{0}, a \in P$. Then we have

$$
\begin{aligned}
f(a) & =f\left(\sum_{k<N} n_{a k} b_{k}\right) \leq \sum_{k<N} n_{a k} f\left(b_{k}\right) \leq \sum_{k<N} n_{a k} c_{k} \\
& =x_{a} \leq \sum_{k<N} n_{a k} g\left(b_{k}\right) \leq g\left(\sum_{k<N} n_{a k} b_{k}\right)=g(a) .
\end{aligned}
$$

- Let $a, b$ be in $P$. Since $n_{a+b, k}=n_{a k}+n_{b k}$ for all $k<N$, we have $x_{a+b}=x_{a}+x_{b}$.

Thus $\left(x_{a}\right)_{a \in Q}$ is a solution of $S_{0}$. But $X$ and $Y$ are nonempty, thus $S$ is bounding. By assumption, $S$ admits a solution $\left(x_{a}\right)_{a \in A^{+}}$. The map $a \mapsto x_{a}$ extends naturally to a homomorphism $h$ from $A$ to $E$. Then $h$ satisfies the required condition.

(b) Let $f, f^{\prime}, g, g^{\prime}$ in $\operatorname{Hom}_{\mathbb{Z}}(A, E)$ be such that $f, f^{\prime} \leq^{+} g, g^{\prime}$. By (a), there exists $h$ in $\operatorname{Hom}_{\mathbb{Z}}(A, E)$ such that $f, f^{\prime} \leq^{+} h \leq^{+} g, g^{\prime}$. Therefore, $\operatorname{Hom}_{\mathbb{Z}}(A, E)$ has the interpolation property. Furthermore, it is immediate (taking componentwise suprema and infima) that $\operatorname{Hom}_{\mathbb{Z}}(A, E)$ is monotone $\sigma$-complete. The conclusion follows from the fact that $\operatorname{Hom}_{\mathbb{Z}}^{*}(A, E)$ is an ideal of $\operatorname{Hom}_{\mathbb{Z}}(A, E)$, directed by Proposition 3.3. 


\section{References}

[1] B. Banaschewski and E. Nelson, Equational compactness in equational classes of algebras, Algebra Universalis 2 (1972), 152-165.

[2] A. Bigard, K. Keimel et S. Wolfenstein, Groupes et anneaux réticulés, Lecture Notes in Math. 608, Springer, 1977.

[3] R. Bradford, Cardinal addition and the axiom of choice, Ann. Math. Logic 3 (1971), 111-196.

[4] C. C. Chang and H. J. Keisler, Model Theory, North-Holland, 1973.

[5] S. Găină, Order topologies in Boolean algebras, Rev. Roumaine Math. Pures Appl. 17 (1972), 243-251.

[6] G. Gierz, K. H. Hofmann, K. Keimel, J. D. Lawson, M. Mislove and D. S. Scott, A Compendium of Continuous Lattices, Springer, 1980.

[7] K. R. Goodearl, Partially Ordered Abelian Groups with the Interpolation Property, Math. Surveys Monographs 20, Amer. Math. Soc., 1986.

[8] K. R. Goodearl, D. E. Handelman and J. W. Lawrence, Affine representations of Grothendieck groups and their applications to Rickart $C^{*}$-algebras and $\aleph_{0}$-continuous rings, Mem. Amer. Math. Soc. 234 (1980).

[9] D. Handelman, D. Higgs and J. Lawrence, Directed abelian groups, countably continuous rings, and Rickart $C^{*}$-algebras, J. London Math. Soc. (2) 21 (1980), 193-202.

[10] J. Mycielski and C. Ryll-Nardzewski, Equationally compact algebras II, Fund. Math. 61 (1968), 271-281.

[11] K. P. S. Bhaskara Rao and R. M. Shortt, Weak cardinal algebras, Ann. New York Acad. Sci. 659 (1992), 156-162.

[12] P. S. Rema, On compact topological lattices, Math. Japon. 9 (1964), 93-98.

[13] R. M. Shortt and F. Wehrung, Common extensions of semigroup-valued charges, J. Math. Anal. Appl. 187 (1994), 235-258.

[14] A. Tarski, Cardinal Algebras, Oxford Univ. Press, New York, 1949.

[15] W. Taylor, Some constructions of compact algebras, Ann. Math. Logic 3 (1971), 395-437.

[16] B. Węglorz, Equationally compact algebras (I), Fund. Math. 59 (1966), 289-298.

[17] -, Completeness and compactness of lattices, Colloq. Math. 16 (1967), 243-248.

[18] F. Wehrung, Injective positively ordered monoids I, J. Pure Appl. Algebra 83 (1992), 43-82.

[19] - Metric properties of positively ordered monoids, Forum Math. 5 (1993), 183-201.

[20] —, Restricted injectivity, transfer property and decompositions of separative positively ordered monoids, Comm. Algebra 22 (1994), 1747-1781.

[21] —, Boolean universes above Boolean models, J. Symbolic Logic 58 (1993), 12191250 .

[22] - A compactness property of Dedekind $\sigma$-complete $f$-rings, Algebra Universalis, to appear.

DÉPARTEMENT DE MATHÉMATIQUES

UNIVERSITÉ DE CAEN

14032 CAEN CEDEX, FRANCE 